\title{
Análisis de la relación entre diferentes variables psicológicas en el contexto deportivo de los futbolistas*
}

\section{Analysis of the Relationship between Different Psychological Variables in the Sporting Environment of Footballers}

Recepción: 08 Agosto 2018 | Aceptación: 03 Noviembre 2019

\author{
ENRIQUe IGLESIAS \\ Universidad de Oviedo, España \\ ORCID: http://orcid.org/0000-0003-0345-7930 \\ José Antonio Cecchini \\ Universidad de Oviedo, España \\ ORCID: http://orcid.org/0000-0003-4098-1029 \\ Marisol Cueli \\ Universidad de Oviedo, España \\ ORCID: http://orcid.org/0000-0002-4662-0534 \\ Paloma González-Castro ${ }^{\mathrm{a}}$ \\ Universidad de Oviedo, España \\ ORCID: http://orcid.org/0000-0001-6685-2933
}

\begin{abstract}
a Autor de correspondencia. Correo electrónico: mgcastro@uniovi.es
\end{abstract}

Para citar este artículo: Iglesias, E., Cecchini, J. A., Cueli, M., \& González-Castro, P. (2019). Análisis de la relación entre diferentes variables psicológicas en el contexto deportivo de los futbolistas. Universitas Psychologica, 18(4), 1-12. https://doi.org/10.11144/ Javeriana.upsy 18-4.ardv

\section{RESUMEN}

En el rendimiento deportivo, influyen variables psicológicas como el clima motivacional, las orientaciones de meta, las necesidades psicológicas básicas del deporte y la cohesión del equipo. Este estudio plantea como objetivo examinar la relación entre este conjunto de variables psicológicas (clima motivacional, la orientación de meta, las necesidades psicológicas básicas del deporte y la cohesión) en varones y mujeres futbolistas. Participaron 175 futbolistas de entre 12 y 26 años $(M=15.59, D E=$ 2.8) que compiten en clubs de diferentes categorías de la Liga Española de Futbol. Todos ellos realizan cuatro cuestionarios dirigidos a valorar el clima motivacional (de maestría y ejecución), las orientaciones de meta (a la tarea o al yo), las necesidades psicológicas (de competencia, autonomía y relación) y la cohesión (de tarea-grupal o de grupo-social). Los análisis de correlación y regresión reflejan relaciones estadísticamente significativas, destacando la relación entre el clima de maestría, la orientación a la tarea y las tres necesidades psicológicas básicas del deporte. Además, la variable con mayor poder predictivo de la satisfacción de las necesidades psicológicas es el clima de maestría. La presencia de un clima motivacional de maestría se relaciona significativamente con otras variables psicológicas positivas para la práctica deportiva.

Palabras clave

clima motivacional; orientaciones de meta; cohesión, fútbol; deporte colectivo.

\section{ABSTRACT}

Psychological variables such as motivational climate, goal orientations, basic psychological sport needs and team cohesion have an influence on sports performance. This study aims at examining the relationships 
among that set of psychological variables (motivational climate, goal orientation, basic psychological sport needs and cohesion) in male and female footballers. Participants were 175 soccer players, aged 12 to $26(M=15.59$, $S D=2.80$ ), competing in different levels of the Spanish Football League. They all took four questionnaires aimed at evaluating the motivational climate (mastery and execution), goal orientations (task and ego-involving), psychological needs (for competence, autonomy and relation) and cohesion (group-task or group-social). The analyses of correlation and regression show significant statistic relations particularly that between mastery climate, task orientation and the three basic psychological needs in sport. Furthermore, the best variable in predicting psychological needs is mastery (involving) climate. The presence of a perceived mastery (involving) motivational climate is significantly related to other positive psychological variables for sport practice.

Keywords

motivational climate; goal orientations; cohesion; soccer; team sport.

Existen diversas características psicológicas relacionadas con los procesos grupales que mejoran la eficacia y el rendimiento en los deportes colectivos y que pueden modificar e influir en el resultado de la competición deportiva (Aspano, Lobato, Leyton, Batista, \& Jiménez, 2016; Kyle, Hernández-Mendo, ReigalGarrido, \& Morales-Sánchez, 2016). Por este motivo, el análisis de las variables psicológicas en el contexto deportivo, constituye una de las áreas de mayor crecimiento en los últimos años (Cecchini, Carriedo, \& Méndez-Giménez, 2019; León-Prados, Fuentes, \& Calvo, 2014).

Entre estas variables psicológicas se encuentra el clima motivacional y sus dos dimensiones, clima de maestría y clima de ejecución, términos introducidos por Ames (1992) para describir el ambiente que crea el entrenador en el contexto deportivo. El clima de maestría (o de tarea), basado en el propio progreso y en la mejora individual se caracteriza porque los profesores, entrenadores o padres promueven valores como la satisfacción, el interés, la diversión y la superación personal (Jaakkola, Yli-Piipari, Barkouris, \& Liukkonen, 2017). Sin embargo, el clima de ejecución, se encuentra asociado con afectividad negativa y sentimientos de presión (González-Cutre, Sicilia, Moreno,
\& Fernández-Balboa, 2009). Este clima de ejecución promueve la comparación social como base del éxito, y las recompensas se establecen de manera pública y basadas en la demostración de un rendimiento superior (Bortoli, Bertollo, Vitali, Filho, \& Robazza, 2015; Jaakkola et al., 2017).

La distinción entre estos dos tipos de clima motivacional resulta pertinente teniendo en cuenta la teoría de la autodeterminación (Cecchini et al., 2019; Deci \& Ryan, 1985; Ryan $\&$ Deci, 2017), en la que se postula que el clima motivacional va a influir en la motivación a través de la satisfacción de tres necesidades psicológicas básicas del deporte, la competencia, la autonomía y las relaciones (Amado et al., 2015; Deci \& Ryan, 2000; Gené \& Latinjak, 2014). La competencia se refiere a la búsqueda de dominio y control sobre las situaciones, de manera que nos sintamos eficaces y capaces; la autonomía, en cambio, se refiere al grado en que los individuos perciben que son la fuente de su propia acción y dirigen su comportamiento y, por último, la relación se define como el grado en que los individuos buscan la interacción y la sensación de pertenencia y conexión con otras personas de su entorno social (Deci \& Ryan, 2000). La teoría de la autodeterminación asume que la satisfacción de estas necesidades favorece el bienestar psicológico y físico, mientras que cuando las necesidades psicológicas se frustran, ello genera malestar y dificulta el crecimiento psicológico de las personas (De Francisco, Arce, Sanchez-Romero, \& Vílchez, 2018; Vallerand \& Verner-Filion, 2013). Al mismo tiempo, estudios como el de Sánchez-Oliva, Leo, Sánchez-Miguel, Amado y García-Calvo (2012) apoyan la relación existente entre la teoría de la autodeterminación y la teoría de las metas de logro, señalando la importancia del clima de maestría sobre la satisfacción de las tres necesidades psicológicas básicas del deporte (competencia, autonomía y relación), la presencia de una motivación más autodeterminada y el desarrollo de comportamientos positivos.

El clima motivacional se relaciona también con las orientaciones de meta de los deportistas a través de la teoría de las metas de logro (Nicholls, 1992). En la teoría de motivación o 
metas al logro, se plantean dos orientaciones de los deportistas en cuanto a su forma de obtener los objetivos. En dicha teoría, se asume la existencia de al menos dos orientaciones de meta, una orientación a la tarea (o a la maestría) en la que se define la competencia en términos de criterios autorreferenciados, y una orientación al yo (al ego o al resultado) que la define utilizando criterios de comparación externa. La relevancia de estas orientaciones de meta, queda reflejada en el deporte en la medida en que alcanzar mejores resultados o lograr maestría en una tarea constituye el objetivo de las personas que están orientadas a la tarea, en contraposición con aquellos orientados al yo (o al ego) que persiguen la aprobación social y realizan atribuciones externas para los resultados (Modroño \& Guillén, 2016; Zubiaur \& Del Riego, 2015). Ambos tipos de orientaciones de meta se pueden dar al mismo tiempo en cierto grado (Saies, Arribas-Galarrag, Cecchini, LuisDe-Cos, \& Otaegui, 2014), incluso la interacción de una alta orientación al yo acompañada de una alta orientación a la tarea puede ser positiva para el rendimiento del deportista (Reyes-Bossio, 2009).

Respecto a la relación existente entre el clima motivacional y la cohesión en el contexto deportivo, existen diversas investigaciones que afirman la existencia de una estrecha relación entre el clima motivacional y la cohesión de equipo (Eys et al., 2013; Gómez-Millán, Vega, \& Gavira, 2017). En el modelo de Carron, Eys y Burke (2007), se detallan cuatro antecedentes principales que intervienen en el desarrollo de la cohesión en el deporte: los factores ambientales, los factores personales, los factores de liderazgo y los factores de equipo. Estos factores se distinguen a su vez en función de dos niveles de interacción; en el primer nivel, se encuentra la integración grupal y la atracción al grupo y en el segundo, están los aspectos de tarea y sociales (Leo, Sánchez-Miguel, Sánchez-Oliva, Amado, \& García-Calvo, 2013). Estos factores en sus niveles de interacción, van a determinar un tipo y un nivel de cohesión que autores como Balaguer, Castillo, Ródenas, Fabra y Duda (2015) han relacionado con el rendimiento deportivo alcanzado.

Finalmente, teniendo en cuenta que Ersöz (2016) pone de manifiesto la necesidad de analizar la relación entre distintas variables en el contexto de la teoría de la motivación autodeterminada, el presente estudio plantea como objetivo analizar la relación entre determinadas variables psicológicas, como el clima motivacional, la orientación de meta, la cohesión y la satisfacción de las necesidades psicológicas básicas del deporte, en un grupo de deportistas que practican fútbol en diferentes categorías. Además, se trató de analizar cómo algunas variables psicológicas como el clima motivacional, las orientaciones de meta y la cohesión predicen la satisfacción de las necesidades psicológicas de competencia, autonomía y relación.

\section{Método}

\section{Participantes}

Participaron en esta investigación 175 futbolistas (110 hombres y 65 mujeres), con edades comprendidas entre los 12 y los 26 años $(M$ $=15.59, \mathrm{DT}=2.8$ ), pertenecientes a equipos de fútbol en diferentes categorías (infantil $n$ $=49$ futbolistas, cadete $n=46$, juvenil $n=28$, regional $n=38$, primera división $n=14)$. La muestra se obtuvo mediante un procedimiento intencional, siguiendo un muestreo por conveniencia.

\section{Instrumentos}

Cuestionario del Clima Motivacional Percibido en el Deporte-2 (PMCSQ-2)

Para medir las percepciones del clima motivacional, se utilizó la versión adaptada al español del Cuestionario del Clima Motivacional Percibido en el Deporte, de Newton, Duda y Yin (2000). Esta versión adaptada (Cecchini, González, López-Prado, \& Brustad, 2005) posee 
una escala que está constituida por dos factores de primer orden, denominados Maestría y Ejecución y seis de segundo orden. En este estudio, se registran los dos factores de primer orden, el clima de maestría (p. ej., "Lo primordial es mejorar"), y clima de ejecución (p. ej., "El entrenador/a solo se fija en los mejores"). La modalidad de respuesta es tipo Likert con puntuaciones que oscilan entre 1 (totalmente en desacuerdo) y 5 (totalmente de acuerdo). En este estudio, se obtuvieron valores alfa de Cronbach de 0.85 para el clima de maestría y de 0.88 para el clima de ejecución.

\section{Cuestionario de Percepción de Éxito}

Para evaluar las disposiciones de meta de logro se empleó el Cuestionario de Percepción de Éxito (Perception of Sucess Questionnaire [POSQ]), elaborado por Roberts y Balagué (1991). El POSQ es una escala de 12 ítems: 6 de orientación de meta a la tarea (p. ej., "Demuestro una clara mejoría personal") y 6 de orientación de meta al yo (p. ej., "soy claramente superior a los demás"). La robustez del POSQ está contrastada en su versión española (Cervelló, Escartí, \& Balagué, 1999). Los participantes respondieron a cada ítem siguiendo la instrucción "Siento éxito en el deporte cuando...". El cuestionario se responde en una escala tipo Likert con puntuaciones que oscilan entre 1 (totalmente en desacuerdo) y 5 (totalmente de acuerdo). En este estudio, se obtuvieron valores alfa de Cronbach de 0.75 para la orientación a la tarea y 0.86 para la orientación al yo.

Escala de las Necesidades Psicológicas Básicas en el Ejercicio (BPNES)

Se utilizó la versión en español de la escala (Moreno-Murcia, Martínez-Galindo, MorenoPérez, Marcos, \& Borges, 2012; Vlachopoulos \& Michailidou, 2006) que está compuesta por 12 ítems, cuatro por cada una de las tres dimensiones: autonomía (p. ej., "tengo la oportunidad de elegir cómo realizar el ejercicio"), competencia (p. ej., "ejecuto eficazmente los ejercicios de mi programa de actividades") y relación (p. ej., "me relaciono de forma muy amistosa con el de compañeros). La modalidad de respuesta es tipo Likert con puntuaciones que oscilan entre 1 (totalmente en desacuerdo) y 5 (totalmente de acuerdo). En la muestra actual los valores alfa de Cronbach oscilan entre 0.71 para autonomía, 0.72 para competencia y 0.74 para relación.

\section{Escala de Cohesión en equipos deportivos}

Se utilizó la adaptación española del Group Environment Questionnaire (Iturbide, Elosua, $\&$ Yanes, 2010). La prueba consta de 18 ítems, distribuidos en cuatro factores: Integración Grupal hacia la Tarea (GI-T; compuesto por cinco ítems, p. ej., "Los componentes del equipo tenemos aspiraciones que no coinciden con el rendimiento del grupo"), Integración Grupal hacia lo Social (GI-S; formado por cuatro ítems, p. ej., "Los componentes del equipo rara vez salimos juntos"), Atracción Individual hacia el Grupo en la Tarea (ATG-T; integrado por cuatro ítems, p. ej., "No estoy satisfecho con el tiempo de juego de que dispongo en el equipo") y Atracción Individual hacia el Grupo en lo Social (ATGS-S; formado por cinco ítems, p. ej., "No disfruto participando en las actividades sociales organizadas por el equipo"). Con base en los resultados obtenidos en la adaptación al español en los que se observó que el modelo bidimensional tarea/social es el que mejor explica la estructura latente de la escala, se agruparon los ítems en dos dimensiones: tarea-grupal (GI$\mathrm{T}+$ ATG-T) y grupo-social (GI-S + ATG-S) . Las respuestas son tipo Likert con puntuaciones que oscilan entre 1 (totalmente en desacuerdo) y 9 (totalmente de acuerdo). En esta escala, dado que los ítems están formulados de manera negativa, puntuaciones altas son indicativas de baja cohesión en cada una de las dimensiones. Los valores alfa de Cronbach en esta muestra resultan de 0.75 para tarea-grupal y 0.71 para la variable grupo-social. 
Procedimiento

Los deportistas cumplimentaron los cuestionarios siguiendo las mismas instrucciones e información sobre el estudio. Se obtuvo el consentimiento informado por parte de los padres y madres o tutores de los deportistas menores de edad. La participación fue voluntaria y toda la información fue tratada de forma confidencial y anónima. Los cuestionarios se cumplimentaron durante una sesión de entrenamiento en la que se solicitó la colaboración rogando sinceridad, reflexión y atención a cada una de las preguntas. La aplicación se llevó a cabo de forma individual o en grupo pequeño. Los datos recogidos se registraron y analizaron utilizando el programa SPSS 19.00 .

\section{Análisis de datos}

Con objeto de estudiar las relaciones que se establecen entre el clima motivacional, la orientación de meta, las necesidades psicológicas y la cohesión, se realizaron análisis descriptivos y análisis de correlaciones bivariadas. Las variables incluidas fueron: clima de maestría y clima de ejecución; orientación a la tarea y orientación al yo; autonomía, competencia y relaciones; tareagrupal y grupo-social.

En un segundo momento, con el objetivo de analizar cómo el clima motivacional, las orientaciones de meta y la cohesión predicen la satisfacción de las necesidades psicológicas de competencia, autonomía y relación, se realizaron análisis de regresión lineal jerárquica. Para ello, se incluyeron como variables dependientes las tres necesidades psicológicas básicas del deporte (competencia, autonomía y relación) y como variables independientes el clima motivacional, las orientaciones de meta y la cohesión de equipo.

\section{Resultados}

\section{Estadísticos descriptivos y correlaciones bivariadas}

Las medias y desviaciones estándar para todas las variables recogidas se presentan en la Tabla 1 . Los resultados del análisis de correlaciones bivariadas indican que el clima de maestría se relaciona negativamente con el clima de ejecución. Sin embargo, la orientación de meta a la tarea lo hace positivamente con la orientación de meta al yo. A su vez, el clima de maestría se relaciona positivamente con la orientación de meta a la tarea y negativamente con la orientación de meta al yo. El clima de ejecución se relaciona positivamente con la orientación de meta al yo, pero no muestra relación significativa con la orientación de meta a la tarea.

Con respecto a las dos variables que miden la cohesión del equipo (tarea-grupal y gruposocial), ambas se relacionan negativamente y de forma significativa con la mayoría de las variables restantes (hay que recordar que valores altos en estas dos variables de cohesión, representan niveles bajos, y viceversa), salvo con el clima de ejecución y la orientación de meta al yo.

Por último, las variables que miden la satisfacción de las necesidades psicológicas, se relacionan positivamente y de manera significativa con el clima de maestría y la orientación de meta a la tarea y negativamente con las restantes (con la excepción de la necesidad de competencia y la orientación de meta al yo en las que la relación es positiva).

\section{Tabla 1}

Análisis descriptivos y correlaciones bivariadas para cada una de las variables incluidas en el estudio

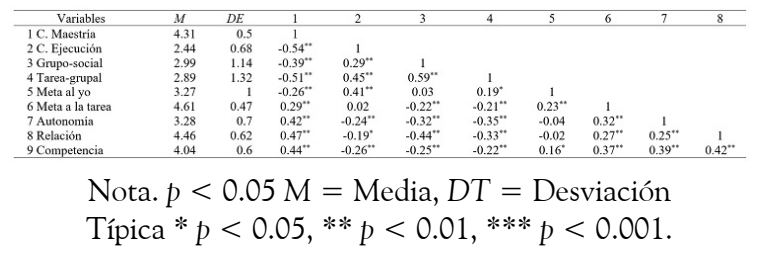


Análisis de regresión jerárquica

Los resultados del análisis de regresión muestran que el clima de maestría es el único que predice positivamente la satisfacción de las tres dimensiones de las necesidades psicológicas básicas del deporte entre las variables analizadas. En el caso de la competencia, el clima de ejecución también presenta un valor predictivo significativo, pero en este caso, negativo.

Del modelo bidimensional tarea/social de la cohesión de equipos deportivos, solo la dimensión grupo-social predice la satisfacción de la necesidad psicológica de relación. Por último, en relación a las orientaciones de meta, ambas (tarea y ego) predicen positivamente la satisfacción básica en el deporte de necesidad de competencia, ninguna la satisfacción de relación social y solamente la orientación a la tarea predice positivamente la autonomía.

\section{Tabla 2}

Análisis de regresión jerárquica tomando como variables dependientes las tres necesidades psicológicas (competencia, relación y autonomía) y como independientes las dimensiones del clima motivacional, de la orientación de meta y de la cohesión de equipo

\begin{tabular}{|c|c|c|c|c|c|c|c|}
\hline \multirow{2}{*}{ Paso } & \multicolumn{3}{|c|}{ Competencia } & \multicolumn{2}{|c|}{ Relación } & \multicolumn{2}{|c|}{ Autonomía } \\
\hline & Predictores & B & $R^{2}$ & B & $R^{2}$ & B & $R^{2}$ \\
\hline $\begin{array}{c}\text { Paso } \\
1 \\
\end{array}$ & $\begin{array}{l}\text { Clima de maestría } \\
\text { Clima de ejecución }\end{array}$ & $\begin{array}{r}0.365 * * * \\
-0.063 \\
\end{array}$ & 0.161 & $\begin{array}{r}0.543 * * * \\
0.078 \\
\end{array}$ & 0.258 & $\begin{array}{r}0.446 * * * * \\
0.001 \\
\end{array}$ & 0.198 \\
\hline & Clima de maestría & $0.331^{* * * *}$ & & $0.422 * * *$ & & $0.358 * * *$ & \\
\hline $\begin{array}{c}\text { Paso } \\
2\end{array}$ & $\begin{array}{l}\text { Clima de ejecución } \\
\text { Grupo-social } \\
\text { Tarea-grupal }\end{array}$ & $\begin{array}{r}-0.05 \\
-0.101 \\
-0.004\end{array}$ & 0.17 & $\begin{array}{r}0.131 \\
-0.306^{* * * *} \\
-0.05\end{array}$ & 0.348 & $\begin{array}{r}0.039 \\
-0.099 \\
-0.138\end{array}$ & 0.231 \\
\hline $\begin{array}{c}\text { Paso } \\
3\end{array}$ & $\begin{array}{l}\text { Clima de maestría } \\
\text { Clima de ejecución } \\
\text { Grupo-social } \\
\text { Tarea-grupal } \\
\text { Orientación tarea } \\
\text { Orientación yo }\end{array}$ & $\begin{array}{r}0.266^{* *} \\
-0.235^{*} \\
-0.015 \\
-0.028 \\
0.254^{* *} \\
0.272^{* *}\end{array}$ & 0.315 & $\begin{array}{r}0.378^{* * * *} \\
0.077 \\
-0.28^{* *} \\
-0.045 \\
0.128 \\
0.035 \\
\end{array}$ & 0.364 & $\begin{array}{r}0.274^{* * *} \\
-0.032 \\
-0.059 \\
-0.131 \\
0.226^{* *} \\
0.003\end{array}$ & 0.273 \\
\hline
\end{tabular}

Nota. $* p<0.05, * * p<0.01, * * * p<0.001$.

\section{Discusión}

La psicología ha desarrollado y consolidado su presencia en todo el espectro de la práctica deportiva, competitiva, profesional y amateur. A lo largo de los años, este crecimiento se ha reflejado en un mayor interés sobre el análisis y el conocimiento de las variables que intervienen en el rendimiento deportivo y que condicionan la ejecución y resultado alcanzado. De ahí, la necesidad de identificar las variables psicológicas que permiten a los deportistas optimizar su comportamiento y rendimiento. En esta línea, este estudio planteó como objetivo analizar la relación entre variables psicológicas como el clima de motivación (de maestría y de ejecución), las orientaciones de meta (a la tarea y al yo), las necesidades psicológicas básicas del deporte (de competencia, autonomía y relación) y la cohesión de equipo (tarea-grupal y grupo-social). Además, se examinó cómo algunas variables psicológicas como el clima motivacional, las orientaciones de meta y la cohesión, predicen la satisfacción de las necesidades psicológicas de competencia, autonomía y relación.

Los resultados obtenidos en el estudio señalan la existencia de relaciones estadísticas de interés entre las variables incluidas, el clima motivacional, la orientación a la tarea, la cohesión y las necesidades psicológicas básicas.

En primer lugar, en el marco de la teoría de las metas de logro sobre el clima motivacional (Newton et al., 2000), se incluyeron las dimensiones del clima, el clima de maestría (implicación en la tarea) y el clima de ejecución (centrado en el yo o en el ego). Los resultados señalaron que ambos tipos de clima correlacionan negativamente en un extremo opuesto de un continuo. En este sentido, dada la correlación negativa entre el clima de maestría y el clima de ejecución encontrada, conviene, en un deporte colectivo como es el fútbol, potenciar el clima de maestría, sobre todo, porque la presencia de un clima de ejecución implicaría la predominancia de objetivos individuales centrados únicamente en el yo. Respecto a la relación entre el clima motivacional y las otras variables incluidas (orientaciones de meta, necesidades psicológicas y cohesión de equipo), también se hallan relaciones estadísticas significativas.

Los resultados de relación entre el clima de motivación y las orientaciones de meta, indican que el clima de maestría se relaciona positivamente con la orientación de meta a la tarea y negativamente con la orientación de meta al yo, en la línea de los resultados aportados por estudios como el de Moreno-Murcia, Cervelló 
y González-Cutre (2010). Estos datos implican que la socialización o el clima generado por el entrenador y el entorno tiene importantes implicaciones sobre la forma de interpretar el éxito y definir la competencia (potenciando metas dirigidas hacia la tarea cuando el clima así lo favorece), tal y como apuntan trabajos como el de Iwasaki y Fry (2016) o Møllerløkken, Lorås y Pedersen (2017). En contraposición, en este estudio, el clima de ejecución no muestra una relación significativa con la orientación de meta a la tarea. La explicación podría relacionarse con que la predisposición personal (centrada en el yo o en el ego) que determina, en gran medida, la probabilidad a priori de adoptar una meta en concreto y de representar un patrón propio de comportamiento. Estas relaciones apuntan nuevamente hacia la necesidad de potenciar en un deporte de equipo como es el fútbol, un clima motivacional de maestría, el cual, se va a relacionar con orientaciones de meta a la tarea por encima de las individuales.

En lo que respecta a la relación entre el clima y las necesidades psicológicas básicas del deporte, la relación es positiva entre las tres necesidades y el clima de maestría y la orientación de meta a la tarea. Sin embargo, las tres necesidades psicológicas básicas se relacionan negativamente con las restantes variables incluidas en el estudio. Este resultado implica que el fomento de un clima de maestría por parte de los entrenadores, va a influir directamente en la satisfacción de las necesidades psicológicas en el deporte, afectando la mejora del bienestar psicológico y físico del deportista, indispensable para su crecimiento psicológico (Vallerand \& Verner-Filion, 2013).

Respecto a las dos variables de cohesión (tarea-grupal grupo-social), se relacionaron negativamente con el clima de ejecución y la orientación al yo, en cambio se vieron favorecidas con el resto de las variables estudiadas. Estas relaciones indican, que un clima de ejecución beneficia escasamente la cohesión del equipo, al contrario que el clima de maestría. De esta forma, el entrenador aparece como una figura promotora de la cohesión del equipo, lo que influirá en un mayor rendimiento del equipo, y en el aumento del compromiso de los deportistas
(Leo, Sánchez-Miguel, Sánchez-Oliva, Amado, \& García-Calvo, 2011).

Por último, en lo que corresponde al análisis de relaciones efectuado en este estudio, se encuentra que las orientaciones de meta a la tarea y al yo correlacionan positivamente. Este resultado, puede adjudicarse a que estas variables poseen un carácter ortogonal, pudiendo presentarse al mismo tiempo en cierto grado (Zarauz, Ruiz-Juan, \& Flores-Allende, 2016). Por su parte, Reyes-Bossio (2009) destaca que una alta orientación al yo acompañada de una orientación a la tarea puede ser positiva para el rendimiento del deportista. Teniendo en cuenta este resultado, conviene resaltar la importancia de promover en la práctica deportiva ambos tipos de orientaciones, dado que la presencia de una orientación al yo (o al ego) se relaciona positivamente con la orientación a la tarea, y de ahí, con mayor cantidad de práctica (García-Mas \& Gimeno, 2008).

Por otro lado, el análisis de regresión permitió concretar qué variables de las estudiadas (clima motivacional, orientaciones de meta o cohesión) predicen las necesidades psicológicas básicas asociadas al deporte. En primer lugar, con respecto al clima motivacional, el clima de maestría es el único de entre las estudiadas que predice positivamente la satisfacción de las tres dimensiones de necesidades psicológicas básicas del deporte (competencia, autonomía y relación). Este dato refuerza los hallazgos de Giménez, Río y Estrada (2013), quienes señalan la importancia de generar un clima motivacional de maestría o tarea, dado que este predice positivamente la satisfacción de las necesidades psicológicas básicas del deporte, con las cuales, siguiendo a Jaakkola, Wang, Soini y Liukkonen (2015), se fomenta de esta forma la adherencia deportiva. Igualmente, resulta de interés que el clima de ejecución (centrado en el ego) presenta un valor predictivo significativo de la competencia, pero en este caso, negativo. Sin embargo, autores como Coterón, Franco, Pérez-Tejero y Sampedro (2013) destacan la relación entre el clima de ejecución y percepción de competencia. Estos autores señalan que en la medida que se alcanzan logros (ego), se va nutriendo la percepción de 
poder realizar las acciones que exige la actividad. También, González-Cutre et al. (2009) resaltan que cuando el clima de ejecución (centrado en el ego) es alto y la percepción de competencia del deportista baja, podrían presentarse patrones desadaptativos, mientras que, si el clima de ejecución y la percepción de competencia son elevados, se presentarían patrones de logro adaptativos. El motivo del resultado alcanzado en el presente estudio podría encontrarse en las edades analizadas, dado que deportistas de entre 12 o 18 años podrían no haber hecho aún una asociación de este tipo.

En segundo lugar, las orientaciones de meta también mostraron poder predictivo sobre las tres necesidades psicológicas básicas del deporte. Los dos tipos de orientaciones de meta, predicen positivamente la satisfacción de la necesidad de competencia, pero no la de relación con los demás, y únicamente la orientación a la tarea predice positivamente la autonomía. Estos resultados pueden ser debidos a la competición a la que se ven sometidos los deportistas (con miembros externos e internos al propio equipo), la cual genera una forma particular de entender las orientaciones de meta a la tarea y al yo (Martínez, Llorca, Tello, \& Núñez, 2016).

En tercer lugar, en la relación entre la cohesión y las necesidades psicológicas básicas del deporte, solo la dimensión grupo-social, de entre las variables analizadas, predice la necesidad de relación con los demás. La dimensión gruposocial evalúa por un lado el interés de los miembros del equipo por juntarse en situaciones ajenas al entrenamiento y los partidos (GI-S), y si los compañeros del equipo configuran uno de los grupos sociales más importantes para ellos (ATG-S). Esta dimensión de grupo-social, encaja entonces en su conjunto con la necesidad psicológica básica de relación, entendida como el grado en que los individuos sienten seguridad de pertenencia y conexión con otras personas de su entorno social (Deci \& Ryan, 2000), por lo tanto, esta dimensión de la cohesión favorecerá esta necesidad psicológica básica dentro del ámbito deportivo.

En este estudio, la dimensión tarea-grupal predice de forma positiva el clima de ejecución y la orientación de meta al yo y negativamente el resto de las variables estudiadas. Hay que resaltar que, la muestra está formada por deportistas pertenecientes a equipos de diferentes categorías, quienes no solo compiten con equipos externos, sino también con miembros del propio equipo o compañeros (para disfrutar de más minutos, más partidos, promoción, etc.). Teniendo esto en cuenta, la promoción de un clima motivacional de ejecución (que implica al ego) no perjudica la cohesión de los jugadores, conscientes de la dinámica en este nivel profesional o de categoría.

Se puede concluir, en este sentido, que los factores ambientales, personales, de equipo y de liderazgo, van a configurar un tipo de orientación de meta, de cohesión y van a condicionar la satisfacción de las necesidades psicológicas básicas en el deporte. Todo ello puede desencadenar una serie de consecuencias, que juegan un papel importante como trasmisoras de valores, actitudes y comportamientos, y que podrían influir en el rendimiento deportivo (Balaguer et al., 2015).

Finalmente, cabe señalar como limitación del presente trabajo, la selección de la muestra de carácter intencional junto con el tamaño muestral. Además, es preciso tener en cuenta a la hora de generalizar los resultados, la dispersión de la muestra, ya que el trabajo incluyó deportistas de diferentes edades, lo que podría influir a la hora de interpretar los resultados. Un mayor número de participantes podría permitir realizar análisis en función de la categoría y concretar las diferencias con base en la edad o al grado o nivel profesional. En el futuro, sería interesante incluir medidas de rendimiento, lo cual, resultaría en una línea futura de interés para profundizar aún más en la relación entre estas variables.

\section{Agradecimientos}

Este trabajo ha sido financiado por un Proyecto $\mathrm{I}+\mathrm{D}+\mathrm{i}$ del Ministerio de Ciencia, Innovación y Universidades de España con referencia PGC2018-097739-B-I00, y por un proyecto regional del Gobierno del Principado de Asturias con referencia FC-GRUPIN-IDI/2018/000199. 


\section{Referencias}

Amado, D., Sánchez-Miguel, P. A., Leo, F. M., Sánchez-Oliva, D., Montero, C., \& GarcíaCalvo, T. (2015). Análisis de los perfiles motivacionales en practicantes de danza: diferencias en función de la modalidad. Revista de Psicología del Deporte, 24(2), 209-216. Recuperado de http://www.redaly c.org/html/2351/235141413002/

Ames, C. (1992). Classrooms: Goals, structures, and student motivation. Journal of Educational Psychology, 84(3), 261. Recuperado de http://hdl.handle.net/1020 1/4432110.1037/0022-0663.84.3.261

Aspano-Carron, M. I., Lobato-Muñoz, S., Leyton-Román, M., Batista, M., \& Jiménez Castuera, R. (2016). Predicción de la motivación en los estadios de cambio de ejercicio más activos. Retos. Nuevas tendencias en Educación Física, Deporte y Recreación, 30, 87-91. Recuperado de http: //hdl.handle.net/10662/6620

Balaguer, I., Castillo, I., Ródenas, L., Fabra, P., \& Duda, J. L. (2015). Los entrenadores como promotores de la cohesión del equipo. Cuadernos de Psicología del Deporte, 15(1), 233-242. Recuperado de http://hdl.handle. net/10201/44321

Bortoli, L., Bertollo, M., Vitali, F., Filho, E., \& Robazza, C. (2015). The effects of motivational climate interventions on psychobiosocial states in high school physical education. Research Quarterly for Exercise and Sport, 86(2), 196-204. https://d oi.org/10.1080/02701367.2014.999189

Carron, A. V., Eys, M. A., \& Burke, S. M. (2007). Team cohesion: Nature, correlates, and development. Social Psychology in Sport, 91-101. Recuperado de http://works.bepres s.com/dr_shauna_burke/31/

Cecchini, J. A., Carriedo, A., \& MéndezGiménez, A. (2019). Testing a circular, feedback model in physical education from self-determination theory. The Journal of Educational Research, 112(4), 473-482. https://doi.org/10.1080/00220671 .2018 .1555788
Cecchini, J. A., González, C., López-Prado, J., \& Brustad, R. J. (2005). Relación del clima motivacional percibido con la orientación de meta, la motivación intrínseca y las opiniones y conductas de fair play. Revista Mexicana de Psicología, 22(2), 469-479. Recuperado de http://www.redalyc.org/arti culo.oa? $\mathrm{id}=243020634010$

Cervelló, E., Escartí, A., \& Balagué, G. (1999). Relaciones entre la orientación de meta disposicional y la satisfacción con los resultados deportivos, las creencias sobre las causas de éxito en el deporte y la diversión con la práctica deportiva. Revista de Psicología del Deporte, 8, 7-21. Recuperado de https://ddd.uab.cat/record/ 63407

Coterón, J., Franco, E., Pérez-Tejero, J., \& Sampedro, J. (2013). Clima motivacional, competencia percibida, compromiso y ansiedad en educación física. Diferencias en función de la obligatoriedad de la enseñanza. Revista de Psicología del Deporte, 22(1), 151-157. https://doi.org/10.5672/ap unts.2014-0983.es.(2018/1).131.04

De Francisco, C., Arce, C., Sánchez-Romero, E. I., \& Vílchez, M. (2018). The mediating role of sport self-motivation between basic psychological needs satisfaction and athlete engagement. Psicothema, 30(4), 421-426. h ttps://doi.org/10.7334/psicothema2018.117

Deci, E. L., \& Ryan, R. M. (1985). The general causality orientations scale: Selfdetermination in personality. Journal of Research in Personality, 19, 109-134. https:// doi.org/10.1016/0092-6566(85)90023-6

Deci, E. L., \& Ryan, R. M. (2000). The "what" and "why" of goal pursuits: Human needs and the self-determination of behaviour. Psychological Inquiry, 11, 227-268. https://d oi.org/10.1207/S15327965PLI1104_01

Ersöz, G. (2016). An examination of motivational regulations, dispositional flow and social physique anxiety among college students for exercise: A self-determination theory approach. College Student Journal, 50 (2), 159-170. Recuperado de https://eric .ed.gov/?id=EJ1104226 
Enrique Iglesias, José Antonio Cecchini, Marisol Cueli, et al.

Eys, M. A., Jewitt, E., Evans, M. B., Wolf, S., Bruner, M. W., \& Loughead, T. M. (2013). Coach-initiated motivational climate and cohesion in youth sport. Research Quarterly for Exercise and Sport, 84, 373-383. https:// doi.org/10.1080/02701367.2013.814909

García-Mas, A., \& Gimeno, F. (2008). La teoría de orientación de metas y la enseñanza de la Educación Física: consideraciones prácticas. Revista Latinoamericana de Psicología, 40(3), 511-522. Recuperado de http://www.redalyc.org/pdf/805/805114 93008.pdf

Gené, P. S., \& Latinjak, A. T. (2014). Relación entre necesidades básicas y autodeterminación en deportistas de élite. Cuadernos de Psicología del Deporte, 14(3), 49-56. Recuperado de http://revistas.um.es /cpd/article/view/211251

Giménez, A. M., Río, J. F., \& Estrada, J. A. C. (2013). Climas motivacionales, necesidades, motivación y resultados en Educación Física. Aula Abierta, 41(1), 63-72. Recuperado de https://dialnet.unirio ja.es/servlet/articulo?codigo $=4097754$

González-Cutre, D., Sicilia, Á., Moreno, J. A., \& Fernández-Balboa, J. M. (2009). Dispositional flow in physical education: Relationships with motivational climate, social goals, and perceived competence. Journal of Teaching in Physical Education, 28(4), 422-440. https://doi.org/10.1123/jtp e.28.4.422

Gómez-Millán, M. R. B., Vega, P. D., \& Gavira, J. F. (2017). Rendimientos deportivos auto y heteropercibidos y cohesión grupal: un estudio exploratorio. Retos Nuevas tendencias en Educación Física, Deporte y Recreación, 31, 103-106. Recuperado de https://recyt.fecyt.es/index.php/retos/art icle/view/43559

Iturbide, L. M., Elosua, P., \& Yanes, F. (2010). Medida de la cohesión en equipos deportivos. Adaptación al español del Group Environment Questionnaire (GEQ). Psicothema, 22(3), 482-488. https://doi.org/ 10.7334/psicothema2014.247
Iwasaki, S., \& Fry, M. (2016). Female adolescent soccer players' perceived motivational climate, goal orientations, and mindful engagement. Psychology of Sport and Exercise, 27, 222-231. https://doi.org/10.10 16/j.psychsport.2016.09.002

Jaakkola, T., Wang, C., Soini, M., \& Liukkonen, J. (2015). Students' perceptions of motivational climate and enjoyment in Finnish physical education: A latent profile analysis. Journal of Sports Science and Medicine, 14(3), 477-483. Recuperado de https://www.ncbi.nlm.nih.gov/pubmed/ 26336332

Jaakkola, T., Yli-Piipari, S., Barkouris, V., \& Liukkonen, J. (2017). Relationships among perceived motivational climate, motivational regulations, enjoyment, and PA participation among Finnish physical education students. International Journal of Sport and Exercise Psychology, 15(3), 273-290. https://doi.org/10.1080/1612197 X.2015.1100209

Kyle, T. L., Hernández-Mendo, A., ReigalGarrido, R. E., \& Morales-Sánchez, V. (2016). Efectos de la actividad física en el autoconcepto y la autoeficacia en preadolescentes. Retos. Nuevas tendencias en Educación Física, Deporte y Recreación, 29, 63-65. Recuperado de https://recyt.fecyt.es /index.php/retos/article/view/36873

Leo, F. M., Sánchez-Miguel, P. A., Sánchez-Oliva, D., Amado, D., \& García-Calvo, T. (2011). Interacción de los niveles de cohesión en el grado de compromiso de jóvenes futbolistas. Movimiento Humano, 1, 13-26. Recuperado de https://dialnet.unirioja.es/servlet/articul o? codigo $=3685998$

Leo, F. M., Sánchez-Miguel, P. A., SánchezOliva, D., Amado, D., \& García-Calvo, T. (2013). El liderazgo y el clima motivacional del entrenador como antecedentes de la cohesión y el rol percibido en futbolistas semiprofesionales. Revista de Psicología del Deporte, 22(2), 361-370. Recuperado de http://www.redalyc.org/pdf/2351/23512 8058002.pdf 
León-Prados, J. A., Fuentes, I., \& Calvo, A. (2014). Relación entre ansiedad estado, autoconfianza percibida y rendimiento en baloncesto. Revista Internacional de Medicina y Ciencias de la Actividad Física y el Deporte, 14(55), 527-543. Recuperado de http://cdeporte.rediris.es/revista/revista 55/artrelacion487.htm

Martínez, L. N., Llorca, J. A. S., Tello, F. P. H., \& Núñez, M. L. (2016). Diferencias de metas entre estudiantes y deportistas: análisis de la invarianza del modelo entre ellos. UniversitasPsychologica, 15(4), 1-11. https:// doi.org/10.11144/Javeriana.upsy15-4.mdee

Modroño, C., \& Guillén García, F. (2016). Motivation and self-concept in windsurfers. Revista de Psicología del Deporte, 25(1), 105-112. Recuperado de http://www.redaly c.org/articulo.oa?id $=235143645014$

Møllerløkken, N., Lorås, H., \& Pedersen, A. (2017). A Comparison of players' and coaches' perceptions of the coach-created motivational climate within youth soccer teams. Frontiers in Psychology, 8(109), 1-10. https://doi.org/10.3389/fpsyg.2017.00109

Moreno-Murcia, J. A., Cervelló, E., \& GonzálezCutre, D. (2010). The achievement goal and self-determination theories as predictors of dispositional flow in young athletes. Anales de Psicología, 26(2), 390-399. Recuperado de http://revistas.um .es/analesps/article/view/109391

Moreno-Murcia, J. A., Martínez-Galindo, C., Moreno-Pérez, V., Marcos, P. J., \& Borges, F. (2012). Confirmation of the Basic Psychological Needs in Exercise Scale (BPNES) with a sample of people who do healthy exercise. Journal of Sports Science EF Medicine, 11(1), 141-146. Recuperado de https://www.ncbi.nlm.nih.gov/pmc/artic les/PMC3737849/

Newton, M., Duda, J. L., \& Yin, Z. (2000). Examination of the psychometric properties of the Perceived Motivational Climate in Sport Questionnaire-2 in a sample of female athletes. Journal of Sports Sciences, 18(4), 275-290. https://doi.org/10.1080/02 6404100365018
Nicholls, J. G. (1992). The general and the specific in the development and expression of achievement motivation. En G. Roberts (Ed.), Motivation in sport and exercise (pp. 59-71). Champaign, IL: Human Kinetics.

Reyes-Bossio, M. (2009). Clima motivacional y orientación de meta en futbolistas peruanos de primera división. Cuadernos de Psicología del Deporte, 9(1), 5-20. Recuperado de http ://revistas.um.es/cpd/article/view/75341

Roberts, G. C., \& Balagué, G. (septiembre, 1991). The development and validation of the Perception of Success Questionnaire. Trabajo presentado en el III Congreso de la Fédération Européenne de Psychologie des Sports et des Activités Corporelles [European Federation of Sport Psychology], Colonia, Alemania.

Ryan, R. M., \& Deci, E. L. (2017). Selfdetermination theory: Basic psychological needs in motivation, development, and wellness. Nueva York: Guilford Press.

Saies, E., Arribas-Galarrag, S., Cecchini, J. A., Luis-De-Cos, I., \& Otaegui, O. (2014). Diferencias en orientaciones de meta, motivación autodeterminada, inteligencia emocional y satisfacción con los resultados deportivos entre piragüistas expertos y novatos. Cuadernos de Psicología del Deporte, 14(3), 21-30. Recuperado de http://revistas .um.es/cpd/article/view/211191

Sánchez-Oliva, D., Leo, F. M., Sánchez-Miguel, P. A., Amado, D., \& García-Calvo, T. (2012). Antecedentes motivacionales de los comportamientos prosociales y antisociales en el contexto deportivo. Revista Internacional de Medicina y Ciencias de la Actividad Física y el Deporte, 12 (46), 253-27. Recuperado de https://dialnet.unir ioja.es/servlet/articulo? codigo $=3958489$

Vallerand, R. J., \& Verner-Filion, J. (2013). Making people's life most worth living: On the importance of passion for positive psychology. Terapia Psicológica, 31(1), 35-48. Recuperado de http://www.redalyc. org/pdf/785/78525710004.pdf

Vlachopoulos, S. P., \& Michailidou, S. (2006). Development and initial validation of 
Enrique Iglesias, José Antonio Cecchini, Marisol Cueli, et al.

a measure of autonomy, competence, and relatedness in exercise: The Basic Psychological Needs in Exercise Scale. Measurement in Physical Education and Exercise Science, 10(3), 179-201. https://doi .org/10.1207/s15327841mpee1003_4

Zarauz, A., Ruiz-Juan, F., \& Flores-Ällende, G. (2016). Modelos predictores de la motivación en corredores de fondo en ruta en función de sus hábitos y entrenamiento. Revista Iberoamericana de Psicología del Ejercicio y el Deporte, 11(2), 185-192. Recuperado de http://www.redalyc.org/htm 1/3111/311145841002/

Zubiaur, M., \& Del Riego, M. (2015). Motivaciones e intereses de practicantes de BMX adolescentes: un estudio piloto. Retos. Nuevas tendencias en Educación Física, Deporte y Recreación, 27(1), 109-113. Recuperado de https://recyt.fecyt.es/index. php/retos/article/view/34358

\section{Notas}

* Artículo de investigación. 